\title{
A numerical shallow-water model for gravity currents for a wide range of density differences
}

\author{
Hiroyuki A. Shimizu*, Takehiro Koyaguchi and Yujiro J. Suzuki
}

\begin{abstract}
Gravity currents with various contrasting densities play a role in mass transport in a number of geophysical situations. The ratio of the density of the current, $\rho_{c}$, to the density of the ambient fluid, $\rho_{\mathrm{a}}$, can vary between $10^{0}$ and $10^{3}$. In this paper, we present a numerical method of simulating gravity currents for a wide range of $\rho_{\mathrm{c}} / \rho_{\mathrm{a}}$ using a shallow-water model. In the model, the effects of varying $\rho_{c} / \rho_{\mathrm{a}}$ are taken into account via the front condition (i.e., factors describing the balance between the driving pressure and the ambient resistance pressure at the flow front). Previously, two types of numerical models have been proposed to solve the front condition. These are referred to here as the Boundary Condition (BC) model and the Artificial Bed (AB) model. The front condition is calculated as a boundary condition at each time step in the $B C$ model, whereas it is calculated by setting a thin artificial bed ahead of the front in the $A B$ model. We assessed the $B C$ and $A B$ models by comparing their numerical results with the analytical results for a simple case of homogeneous currents. The results from the BC model agree well with the analytical results when $\rho_{\mathrm{c}} / \rho_{\mathrm{a}} \lesssim 10^{2}$, but the model tends to overestimate the speed of the front position when $\rho_{c} / \rho_{\mathrm{a}} \gtrsim 10^{2}$. In contrast, the AB model generates good approximations of the analytical results for $\rho_{\mathrm{c}} / \rho_{\mathrm{a}} \gtrsim 10^{2}$, given a sufficiently small artificial bed thickness, but fails to reproduce the analytical results when $\rho_{\mathrm{c}} / \rho_{\mathrm{a}} \lesssim 10^{2}$. Therefore, we propose a numerical method in which the BC model is used for currents with $\rho_{\mathrm{c}} / \rho_{\mathrm{a}} \lesssim 10^{2}$ and the AB model is used for currents with $\rho_{\mathrm{c}} / \rho_{\mathrm{a}} \gtrsim 10^{2}$.
\end{abstract}

Keywords: Gravity currents, Numerical model, Shallow-water model, Front condition

\section{Introduction}

Gravity currents are flows driven by density differences between the current and the ambient fluid. In geophysical settings, there are many types of high-Reynolds-number (typically $\gtrsim 10^{3}$ ) gravity currents that show a wide range of density ratios $\left(\rho_{\mathrm{c}} / \rho_{\mathrm{a}}\right.$, where $\rho_{\mathrm{c}}$ and $\rho_{\mathrm{a}}$ are the densities of the current and ambient fluid, respectively), such as debris flows $\left(\rho_{\mathrm{c}} / \rho_{\mathrm{a}} \sim 10^{3}\right.$; e.g., Iverson 1997), turbidity currents $\left(\rho_{\mathrm{c}} / \rho_{\mathrm{a}} \sim 10^{0}\right.$; e.g., Meiburg and Kneller 2010), and pyroclastic density currents $\left(\rho_{\mathrm{c}} / \rho_{\mathrm{a}}=10^{0}-10^{1}\right.$ in the overlying parts and $\rho_{\mathrm{c}} / \rho_{\mathrm{a}}=10^{2}-10^{3}$ in the underlying parts; e.g., Branney and Kokelaar 2002; Breard et al. 2016; Nield and Woods 2004). For the two extreme cases of $\rho_{\mathrm{c}} / \rho_{\mathrm{a}} \sim 10^{0}$ and $10^{3}$, the fluid dynamical features of gravity currents (e.g., the shape of the interface and the propagation of the flow front) have been studied in detail

*Correspondence: s-hiro@eri.u-tokyo.ac.jp

Earthquake Research Institute, The University of Tokyo, 1-1-1 Yayoi, 113-0032 Bunkyo-ku, Tokyo, Japan using experimental investigations (e.g., Marino et al. 2005; Martin and Moyce 1952; Dressler 1954; Rottman and Simpson 1983), numerical investigations (e.g., Cantero et al. 2007; Ooi et al. 2009), and theoretical modeling (e.g., Benjamin 1968; Hogg and Pritchard 2004; Huppert and Simpson 1980; Stoker 1992; Ungarish and Zemach 2005). For intermediate density ratios $\left(10^{0}<\rho_{\mathrm{c}} / \rho_{\mathrm{a}}<10^{3}\right)$, there have been some previous studies (e.g., Birman et al. 2005; Bonometti et al. 2011; Gröbelbauer et al. 1993; Hallworth and Huppert 1998; Härtel et al. 2000; Ungarish 2007), but the dynamics of gravity currents within this density range is less well understood than that of the extreme cases.

The purpose of this study is to develop a numerical model of gravity currents for a wide range of $\rho_{\mathrm{c}} / \rho_{\mathrm{a}}$ based on a shallow-water model. The shallowwater model is an efficient mathematical model that captures the essential features of the vertically averaged motion of gravity currents with free surfaces (see 
Ungarish 2009 for an extensive review). For simple initial and boundary conditions, analytical solutions of the shallow-water model for propagating gravity currents are available for a wide range of $\rho_{\mathrm{c}} / \rho_{\mathrm{a}}$ (Ungarish 2007), and these analytical solutions have been verified by experimental measurements and direct numerical simulations using the Navier-Stokes equation (Bonometti and Balachandar 2010; Ungarish 2007). However, geophysical conditions of interest generally have rather complex initial and boundary conditions, so such analytical solutions are not always available. A numerical model that is applicable for complex initial and boundary conditions is highly desirable for simulations of gravity currents for a wide range of $\rho_{\mathrm{c}} / \rho_{\mathrm{a}}$.

This study is particularly concerned with a numerical treatment of the flow front of gravity currents. In the following sections, we formulate the mathematical problem and show that the numerical treatment at the flow front is key to correctly solving the dynamics of gravity currents for a wide range of $\rho_{\mathrm{c}} / \rho_{\mathrm{a}}$ within the framework of the shallow-water model. We also assess previous numerical methods that have been used to calculate the behavior of the flow front by comparing numerical and analytical results, and we propose a numerical method to simulate the dynamics of gravity currents for a wide range of $\rho_{\mathrm{c}} / \rho_{\mathrm{a}}$ under various geophysical conditions. Finally, as a geophysical application of our results, we develop a numerical model of a pyroclastic density current with strong density stratification.

\section{Methods}

\section{Formulation}

We consider a planar, inviscid, incompressible, immiscible gravity current of density $\rho_{\mathrm{c}}$ in a deep ambient fluid of density $\rho_{\mathrm{a}}$, as shown in Fig. 1 . The current propagates along a smooth horizontal bottom in the positive $x^{*}$ direction in time $t^{*}$, and gravitational acceleration $g$ acts in the negative $z^{*}$ direction, where asterisks denote dimensional variables. The propagating current is initially stationary in a reservoir of length $x_{0}$ and height $h_{0}$, and propagation

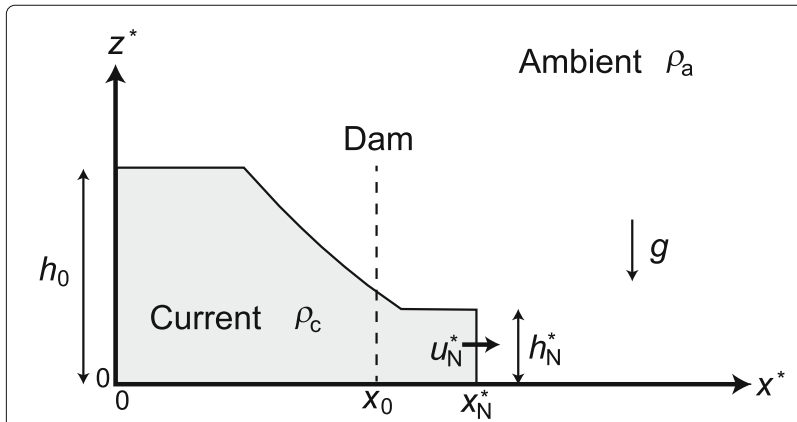

Fig. 1 Schematic of the gravity current released from a dam in a deep ambient fluid occurs after a dam at $x^{*}=x_{0}$ is rapidly removed at $t^{*}=0$. The boundary at $x^{*}=0$ is a rigid wall. The flow front at $x^{*}=x_{\mathrm{N}}^{*}\left(t^{*}\right)$ is affected by the resistance of the ambient fluid, where $\mathrm{N}$ denotes the front. This problem is referred to as the "dam-break problem" (e.g., Ungarish 2009), and is a simple geophysical scenario.

We assume that the current is shallow, with $h_{0} / x_{0} \ll 1$, and is in hydrostatic equilibrium in the vertical direction (i.e., the shallow-water approximation). In the shallowwater approximation, we can obtain the vertically averaged conservation equations of mass and momentum for the flow interior $x^{*}<x_{\mathrm{N}}^{*}$ (e.g., Ungarish 2007) as follows:

$$
\begin{aligned}
& \frac{\partial h^{*}}{\partial t^{*}}+\frac{\partial}{\partial x^{*}}\left(u^{*} h^{*}\right)=0, \\
& \frac{\partial}{\partial t^{*}}\left(u^{*} h^{*}\right)+\frac{\partial}{\partial x^{*}}\left(u^{* 2} h^{*}+\frac{1}{2} \frac{\rho_{\mathrm{c}}-\rho_{\mathrm{a}}}{\rho_{\mathrm{c}}} g h^{* 2}\right)=0,
\end{aligned}
$$

where $h(x, t)$ is the local height and $u(x, t)$ is the local horizontal velocity.

At the flow front $x^{*}=x_{\mathrm{N}}^{*}\left(t^{*}\right)$, the kinematic condition $\left(\mathrm{d} x_{\mathrm{N}}^{*} / \mathrm{d} t^{*}=u_{\mathrm{N}}^{*}\right)$ and the mass and momentum equations should be taken into account. In addition, to describe realistic gravity current dynamics, we must consider a quasi-steady balance between the buoyancy pressure driving the current front $\left(\sim\left(\rho_{\mathrm{c}}-\rho_{\mathrm{a}}\right) g h_{\mathrm{N}}^{*}\right)$ and the resistance pressure caused by the acceleration of the ambient fluid around the front $\left(\sim \rho_{\mathrm{a}} u_{\mathrm{N}}^{* 2}\right)$. This condition is known as the front condition, and can be written as follows (e.g., Ungarish 2007):

$$
u_{\mathrm{N}}^{*}=F r \sqrt{\frac{\rho_{\mathrm{c}}-\rho_{\mathrm{a}}}{\rho_{\mathrm{a}}} g h_{\mathrm{N}}^{*}} \quad \text { at } \quad x^{*}=x_{\mathrm{N}}^{*}\left(t^{*}\right),
$$

where $F r$, which is an imposed frontal Froude number, is assumed to be a constant of order $10^{0}$ (e.g., $\sqrt{2}$; Benjamin 1968).

Here, using $x_{0}$ as the length scale and $h_{0}$ as the height scale, we rewrite all dimensional variables to dimensionless variables as follows:

$$
x=x^{*} / x_{0}, \quad h=h^{*} / h_{0}, \quad u=u^{*} / U, \quad t=t^{*} / T,
$$

with

$$
U=\sqrt{\frac{\rho_{\mathrm{c}}-\rho_{\mathrm{a}}}{\rho_{\mathrm{c}}} g h_{0}}, \quad T=x_{0} / U .
$$

Applying this scaling to Eqs. (1)-(3), we obtain

$$
\begin{aligned}
& \frac{\partial}{\partial t} \boldsymbol{q}+\frac{\partial}{\partial x} \boldsymbol{f}=\mathbf{0} \\
& u_{\mathrm{N}}=F r \sqrt{\rho_{\mathrm{c}} / \rho_{\mathrm{a}}} \sqrt{h_{\mathrm{N}}} \quad \text { at } \quad x=x_{\mathrm{N}}(t)
\end{aligned}
$$

with

$$
\boldsymbol{q}=\left(\begin{array}{c}
h \\
u h
\end{array}\right) ; \quad \boldsymbol{f}=\left(\begin{array}{c}
u h \\
u^{2} h+\frac{1}{2} h^{2}
\end{array}\right)
$$

Note that the density ratio $\rho_{\mathrm{c}} / \rho_{\mathrm{a}}$ is included only in the front condition (7). Hence, to capture the effects of $\rho_{\mathrm{c}} / \rho_{\mathrm{a}}$, 
it is important to calculate the front condition correctly (Ungarish 2007).

The behavior of the analytical solutions for the above equations depends on $\rho_{\mathrm{c}} / \rho_{\mathrm{a}}$ (Fig. 2; Ungarish 2007). The analytical solutions of the dam-break problem consist of an initial "slumping" stage and a subsequent "self-similar" stage (Fig. 2a; e.g., Hogg 2006). During the slumping stage, the front moves with a constant speed and height. During this stage, an initial backward-propagating rarefaction wave arises from the rapidly removed dam, and then a wave arises from the reflection of this rarefaction wave at the back wall $x=0$ at $t=1$. The slumping stage continues until the front is caught by this reflection wave. After the slumping stage, the solution is asymptotic to a self-similar solution as time tends to infinity (i.e., the selfsimilar stage). During this stage, the velocity and height of the front decrease with time. The dependence of the solution on $\rho_{\mathrm{c}} / \rho_{\mathrm{a}}$ is clearly observed in the behavior of the flow front. When $\rho_{\mathrm{c}} / \rho_{\mathrm{a}} \sim 10^{0}$, the front height $h_{\mathrm{N}}$ is on the order of $10^{-1}$ during the slumping stage and in the early self-similar stage (Fig. 2a). On the other hand, when $\rho_{\mathrm{c}} / \rho_{\mathrm{a}} \sim 10^{3}, h_{\mathrm{N}}$ is much smaller than $10^{-1}$, even from the beginning, the front velocity $u_{\mathrm{N}}$ is substantially greater than $u_{\mathrm{N}}$ for $\rho_{\mathrm{c}} / \rho_{\mathrm{a}} \sim 10^{0}$ (Fig. 2b). These differences can be interpreted as follows: the momentum lost due to the resistance of the ambient fluid at the front becomes less significant with respect to the momentum of the current as $\rho_{\mathrm{c}} / \rho_{\mathrm{a}}$ increases. We aim to numerically reproduce these features of the analytical solution below.

\section{Numerical methods}

In this study, we developed a numerical method for modeling gravity currents for a wide range of $\rho_{\mathrm{c}} / \rho_{\mathrm{a}}$ by discretizing the dimensionless mass and momentum conservation equations (Eqs. (6) and (8)). As these equations are nonlinear and hyperbolic, shocks may develop in the currents. Consequently, we used a finite volume method with shock-capturing capability (e.g., LeVeque 2002; Toro 2001). The finite volume method updates a piecewise constant function $\boldsymbol{Q}_{i}^{n}$ that approximates the average value of the solution $\boldsymbol{q}$ in each grid cell $i$ at time step $n$, using the expression

$$
\boldsymbol{Q}_{i}^{n+1}=\boldsymbol{Q}_{i}^{n}-\frac{\Delta t}{\Delta x}\left(\boldsymbol{F}_{i+1 / 2}-\boldsymbol{F}_{i-1 / 2}\right)
$$

where $\Delta x$ is the constant cell length and $\Delta t$ is the time interval. $\boldsymbol{F}_{i+1 / 2}$, which is the intercell flux between cells $i$ and $i+1$, is obtained by using an exact Riemann solver or an approximate Riemann solver, such as the Roe scheme (e.g., LeVeque 2002; Toro 2001). The time interval $\Delta t$ is a
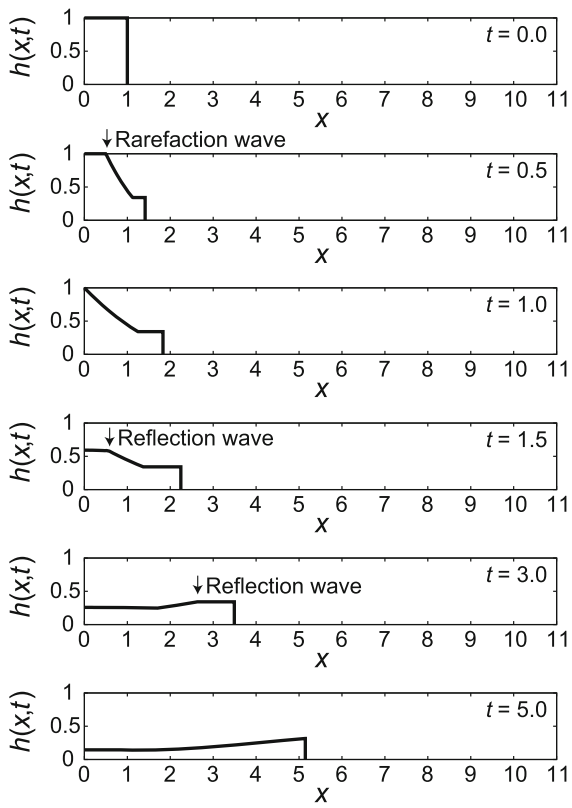

b
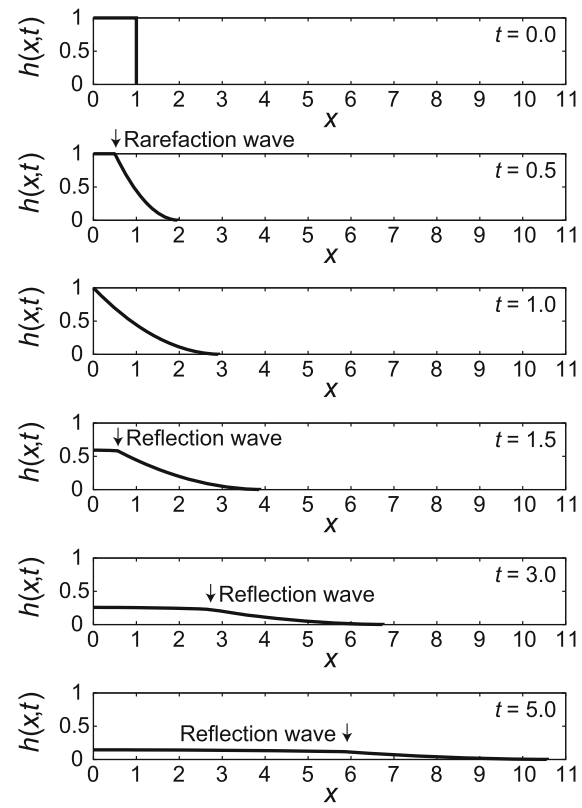

Fig. 2 Analytical solutions of $h(x, t)$ for the dam-break problem. Here, $F r=\sqrt{2}$ (Benjamin 1968) is used. a $\rho_{\mathrm{c}} / \rho_{\mathrm{a}}=1.01 . \mathbf{b} \rho_{\mathrm{c}} / \rho_{\mathrm{a}}=1000$. In $(\mathbf{a})$, the currents at $t=0.5,1.0,1.5$, and 3.0 are in the slumping stage, and the current at $t=5.0$ is in the self-similar stage. The initial backward-propagating rarefaction wave arising from the rapidly removed dam travels toward the back wall $x=0$ (see the profile at $t=0.5$ ), reaching the wall at $t=1.0$. Then, a wave arises from the reflection of the rarefaction wave and travels toward the front (see the profiles at $t=1.5$ and 3.0 ). After the front is caught by this reflection wave, the current is in the self-similar stage (see the profile at $t=5.0$ ). In (b), all the currents are in the slumping stage. In this case (i.e., $\rho_{\mathrm{c}} / \rho_{\mathrm{a}}=1000$ ), the slumping stage continues until $t \sim 226$ (see Hogg 2006 for details) 
limited by the Courant-Friedrichs-Lewy condition (e.g., LeVeque 2002; Toro 2001).

As mentioned above, if we are to capture the effects of $\rho_{\mathrm{c}} / \rho_{\mathrm{a}}$, it is important to calculate the front condition (7) correctly. Previously, two types of numerical models have been proposed to calculate the front condition. In one, the front condition is calculated as a boundary condition at each time step (e.g., Ungarish 2009). We refer to this model as the Boundary Condition (BC) model (Fig. 3a). In the other, the front condition is calculated by setting a thin artificial bed ahead of the front (e.g., Toro 2001). We refer to this as the Artificial Bed ( $A B)$ model (Fig. 3b). In the $A B$ model, the resistance of the ambient fluid at the flow front is modeled by the reaction of the force pushing the artificial bed at the flow front. These models will be described below.

\section{Boundary Condition (BC) model}

In the BC model, three quantities at the flow front $\left(x_{\mathrm{N}}\right.$, $h_{\mathrm{N}}$, and $u_{\mathrm{N}}$ ) are calculated as boundary conditions of the current from the three equations (mass and momentum conservation equations and front condition) at each time step. In the present numerical method, because we apply a fixed spatial coordinate with constant $\Delta x$, the front position $x=x_{\mathrm{N}}(t)$ generally does not coincide with the margins of the grid cells. We therefore define the cell that includes the front as the front cell $(i=F C(t)$, where $F C(t)$ is an integer), and the width of the region that the current occupies in the front cell as $\Delta x_{F C}(t)\left(0 \leq \Delta x_{F C}(t)<\Delta x\right.$; see Fig. 4). Using $F C(t)$ and $\Delta x_{F C}(t)$, we can write the front position as

$$
x_{\mathrm{N}}(t)=(F C(t)-1) \Delta x+\Delta x_{F C}(t) .
$$

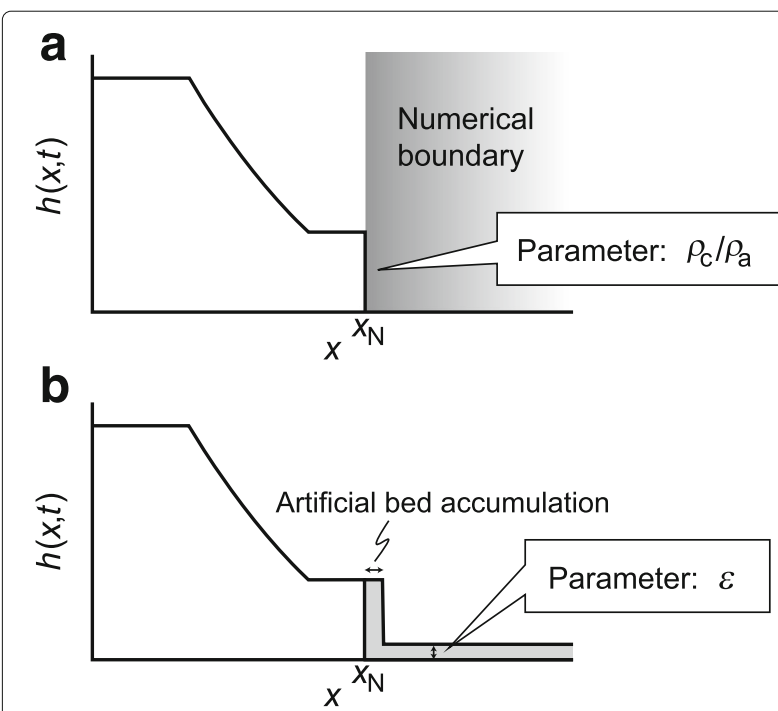

Fig. 3 Schematics of the numerical models used to calculate the front condition. a Boundary Condition (BC) model. b Artificial Bed (AB) model
The values of $h_{\mathrm{N}}$ and $u_{\mathrm{N}}$ are approximated by the values of $h$ and $u$ at the front cell (i.e., $h_{F C}$ and $u_{F C}$ ).

When the kinematic condition $\left(\mathrm{d} x_{\mathrm{N}} / \mathrm{d} t=u_{\mathrm{N}}\right)$ is taken into account, the discretized equations for mass and momentum conservation at the flow front are given by

$$
\Delta x_{F C}^{n+1} h_{F C}^{n+1}=\Delta x_{F C}^{n} h_{F C}^{n}+\Delta t f_{1}
$$

and

$\Delta x_{F C}^{n+1}(u h)_{F C}^{n+1}=\Delta x_{F C}^{n}(u h)_{F C}^{n}+\Delta t\left(f_{2}-\frac{1}{2}\left(h_{F C}^{n+1}\right)^{2}\right)$,

respectively, where $\left(f_{1}, f_{2}\right)^{\mathrm{T}}$ represents the intercell flux $\boldsymbol{F}_{F C-1 / 2}$. From the front condition (i.e., Eq. (7)), we obtain

$$
\frac{(u h)_{F C}^{n+1}}{h_{F C}^{n+1}}=\operatorname{Fr} \sqrt{\rho_{\mathrm{c}} / \rho_{\mathrm{a}}} \sqrt{h_{F C}^{n+1}} .
$$

Solving these three equations analytically (e.g., using Ferrari's method for the solution of the quartic equation) or numerically (e.g., using the Newton-Raphson iteration method), we obtain $h_{F C}^{n+1}, u_{F C}^{n+1}$, and $\Delta x_{F C}^{n+1}$, and hence, $h_{\mathrm{N}}, u_{\mathrm{N}}$, and $x_{\mathrm{N}}$ at each time step.

\section{Artificial Bed (AB) model}

In the $A B$ model, the conservation equations (Eqs. (6) and (8)) are numerically solved using a shock-capturing method for not only the interior, but also the outside of the current by a priori setting a thin artificial bed ahead of the front. Through this numerical procedure, the flow front is generated as the flow following a shock formed ahead of the front without any additional calculation (see Fig. $3 b)$. In this model, the thickness of the artificial bed ( $\varepsilon$ in Fig. $3 \mathrm{~b}$ ) is the parameter that controls the front condition (i.e., the values of $h_{\mathrm{N}}$ and $u_{\mathrm{N}}$ for different values of $\rho_{\mathrm{c}} / \rho_{\mathrm{a}}$; see section 10.8 in Toro 2001).

Here, we analytically determined the relationship between $\varepsilon$ and $\rho_{\mathrm{c}} / \rho_{\mathrm{a}}$, as well as that between $u_{\mathrm{N}}$ and $\varepsilon$, on the basis of the analytical solution for the slumping stage of the dam-break problem (e.g., LeVeque 2002; Toro 2001; Ungarish 2009). The initial conditions are $h=1$ and $u=0$ in the domain $0 \leq x \leq 1$, and $h=\varepsilon$ and $u=0$ in the domain $x>1$, at $t=0$. Let us consider the time evolution of the current before the rarefaction wave reaches the back wall $x=0$ (i.e., $0<t \leq 1$ ).

For hyperbolic equations such as those used in the present system (i.e., Eqs. (6) and (8)), the relationships between the variables (i.e., $h$ and $u$ ) on the characteristics $c_{ \pm}=u \pm \sqrt{h}$ are represented as follows:

$$
\Gamma_{ \pm}=u \pm 2 \sqrt{h}=\text { const } \quad \text { on } \quad \frac{\mathrm{d} x}{\mathrm{~d} t}=c_{ \pm}
$$

where $\Gamma_{ \pm}$are the "Riemann Invariants". Considering that $c_{+}$characteristics from the domain with one initial con- 


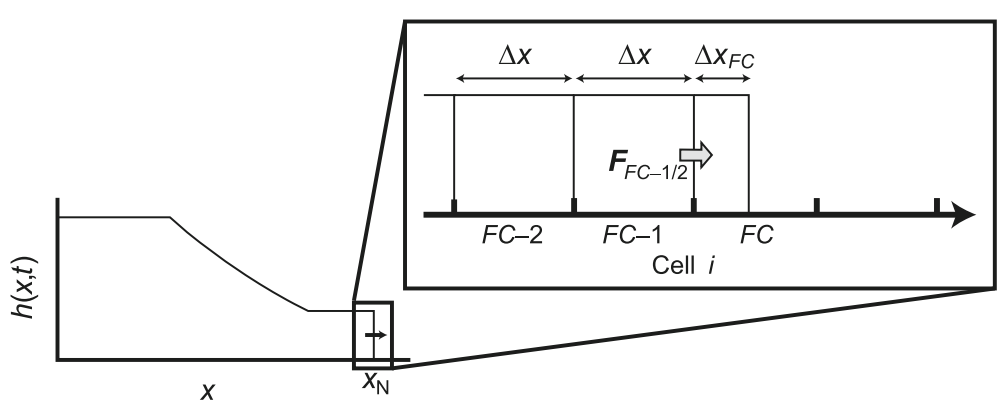

Fig. 4 Schematic of the computational domain of the BC model

dition $(h=1, u=0)$ enter the front domain $\left(h=h_{\mathrm{N}}\right.$, $u=u_{\mathrm{N}}$ ), we can obtain

$$
u_{\mathrm{N}}=2\left(1-\sqrt{h_{\mathrm{N}}}\right)
$$

from Eq. (14). The equation provides the relationship between $h=h_{\mathrm{N}}$ and $u=u_{\mathrm{N}}$ inside the current.

On the other hand, when an artificial bed with $h=\varepsilon$ and $u=0$ is set, a shock wave traveling with speed $S$ occurs ahead of the front. Across this shock wave, the RankineHugoniot condition,

$$
\boldsymbol{f}\left(\boldsymbol{q}_{\mathrm{R}}\right)-\boldsymbol{f}\left(\boldsymbol{q}_{\mathrm{L}}\right)=S\left(\boldsymbol{q}_{\mathrm{R}}-\boldsymbol{q}_{\mathrm{L}}\right)
$$

should hold. Here, the subscript $\mathrm{R}$ denotes the state on the right side of the shock and $\mathrm{L}$ denotes the state on the left side. From Eq. (16), we obtain the state of the front domain behind the shock (i.e., the relationship between $h=h_{\mathrm{N}}$ and $\left.u=u_{\mathrm{N}}\right)$ as

$$
u_{\mathrm{N}}=\left(h_{\mathrm{N}}-\varepsilon\right) \sqrt{\frac{1}{2}\left(\frac{h_{\mathrm{N}}+\varepsilon}{h_{\mathrm{N}} \varepsilon}\right)},
$$

and the shock speed as

$$
S=\sqrt{\frac{1}{2} \frac{\left(h_{\mathrm{N}}+\varepsilon\right) h_{\mathrm{N}}}{\varepsilon}} .
$$

Eliminating $h_{\mathrm{N}}$ from Eqs. (15), (17), and (18), we obtain $u_{\mathrm{N}}$ and $S$ as a function of $\varepsilon$ (Fig. 5a). Using the front condition (7) as well as these equations, we also obtain the relationship between the artificial bed thickness $\varepsilon$ and the density ratio $\rho_{\mathrm{c}} / \rho_{\mathrm{a}}$ (Fig. 5b) as

$$
\begin{aligned}
& \left(1-\frac{2}{F r \sqrt{\rho_{\mathrm{c}} / \rho_{\mathrm{a}}}+2}\right) \frac{4 \sqrt{2 \varepsilon}}{F r \sqrt{\rho_{\mathrm{c}} / \rho_{\mathrm{a}}}+2} \\
& =\left\{\left(\frac{2}{F r \sqrt{\rho_{\mathrm{c}} / \rho_{\mathrm{a}}}+2}\right)^{2}-\varepsilon\right\} \sqrt{\left(\frac{2}{F r \sqrt{\rho_{\mathrm{c}} / \rho_{\mathrm{a}}}+2}\right)^{2}+\varepsilon} .
\end{aligned}
$$

Note that because we use Eq. (15) here, these relationships (Fig. 5) are in the slumping stage.

In Fig. 5 a, $S$ is larger than the front velocity, $u_{\mathrm{N}}$, because of the accumulation of the artificial bed at the flow front (see Fig. 3b). This deviation of $S$ from $u_{\mathrm{N}}$ is substantial for $\varepsilon \gtrsim 10^{-3}$. This implies that the position of the shock does not always approximate the flow front. If we are to extract the correct position of the flow front, we must calculate an advection equation for a passive tracer concentration, $\phi(\phi=1$ for $0 \leq x \leq 1$, and $\phi=0$ for $x>1$, at $t=0)$ :

$$
\frac{\partial \phi}{\partial t}+u \frac{\partial \phi}{\partial x}=0
$$

after solving the equations of fluid motion (see section 13.12 in LeVeque 2002 for details).

\section{Results and discussion}

In this section, we compare the numerical results obtained from the $\mathrm{BC}$ and $\mathrm{AB}$ models with the analytical results, and assess the applicability of these models. Subsequently, as a geophysical application of our results, we develop a numerical model of pyroclastic density currents.

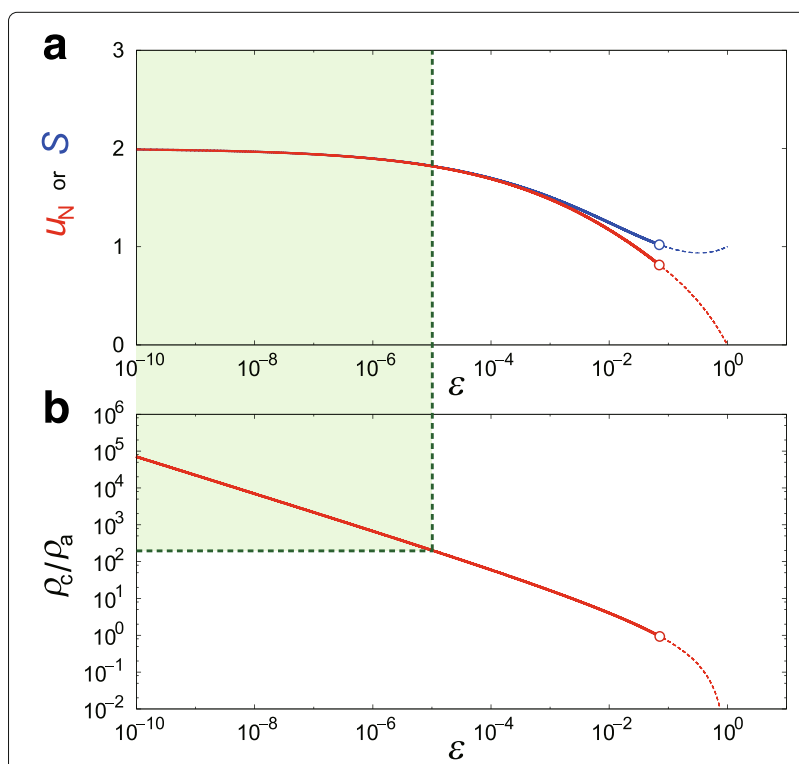

Fig. 5 Analytical solutions for the $A B$ model during the slumping stage. a Front velocity $u_{N}$ (red curve) and shock speed $S$ (blue curve), as functions of $\varepsilon$. b Relationship between $\varepsilon$ and $\rho_{c} / \rho_{a}$, in which $F r=\sqrt{2}$ (Benjamin 1968) is used. Dashed curves represent the solutions for $\rho_{\mathrm{c}} / \rho_{\mathrm{a}}<1$ 


\section{Comparison of analytical and numerical results}

Figure 6 shows the numerical results from the BC model along with the analytical results for the cases of $\rho_{\mathrm{c}} / \rho_{\mathrm{a}}=$ 1.01 (a) and 1000 (b). The numerical results for $\rho_{\mathrm{c}} / \rho_{\mathrm{a}}=$ 1.01 agree well with the analytical results from the early slumping stage to the late self-similar stage. The numerical results for $\rho_{\mathrm{c}} / \rho_{\mathrm{a}}=1000$ also appear to agree with the analytical results, but the speed of the front position, $\dot{x}_{\mathrm{N}}$, shows a numerical oscillation that is not observed in the analytical result (Fig. 7a). In particular, in the initial stage $\left(t \lesssim 0.0002\right.$ in Fig. 7a), $\dot{x}_{\mathrm{N}}$ tends to be overestimated. These oscillation and overestimation are caused by the assumption that the values of $h_{F C}$ and $u_{F C}$ are uniform across the width of the front cell $\Delta x_{F C}$ in the present numerical method at first-order accuracy. For a large $\rho_{\mathrm{c}} / \rho_{\mathrm{a}}$, because $h_{\mathrm{N}}$ has a small value, the value of $\Delta x_{F C}$ tends to be overestimated when a constant $h_{F C}$ is assumed (Fig. 7b). We suggest, therefore, that the BC model is favorable for simulating gravity currents with relatively low $\rho_{\mathrm{c}} / \rho_{\mathrm{a}}$.

Figure 8 shows the numerical results from the $A B$ model along with the analytical results. In these calculations, the values of $\varepsilon$ for given values of $\rho_{\mathrm{c}} / \rho_{\mathrm{a}}$ are set based on the relationship of Eq. (19) (see Fig. 5b). In Fig. 8b, the numerical results for $\rho_{\mathrm{c}} / \rho_{\mathrm{a}}=1000\left(\varepsilon=4.58 \times 10^{-7}\right)$ agree well with the analytical results. The numerical oscillations observed in the $\mathrm{BC}$ model do not occur with the $\mathrm{AB}$ model (Fig. 7a). In Fig. 8a, on the other hand, the numerical results for $\rho_{\mathrm{c}} / \rho_{\mathrm{a}}=1.01\left(\varepsilon=6.58 \times 10^{-2}\right)$ agree well with the analytical results only during the slumping stage ( $t \lesssim 4.5$ ), but deviate from the analytical results during the self-similar stage $(t \gtrsim 4.5)$. This agreement during the slumping stage and deviation during the selfsimilar stage occurs because $\varepsilon$ is set using the analytical relationship (Eq. (19)) for the slumping stage of the dambreak problem. During the slumping stage, $h_{\mathrm{N}}$ and $u_{\mathrm{N}}$ are constant so that $\varepsilon$ based on Eq. (19) provides the correct front condition. During the self-similar stage, on the other hand, the driving pressure, and hence $h_{\mathrm{N}}$ and $u_{\mathrm{N}}$, decrease with time; therefore, the assumed value of $\varepsilon$ is no longer consistent with the front condition Eq. (7).

The good agreement in the results of the $\mathrm{AB}$ model for $\rho_{\mathrm{c}} / \rho_{\mathrm{a}}=1000$ reflects the fact that the dynamics of the gravity current becomes insensitive to the front condition for large values of $\rho_{\mathrm{c}} / \rho_{\mathrm{a}}$. In Fig. $5 \mathrm{~b}, \varepsilon$ approaches 0 as $\rho_{\mathrm{c}} / \rho_{\mathrm{a}}$ increases. In the limit as $\rho_{\mathrm{c}} / \rho_{\mathrm{a}} \rightarrow \infty$ and $\varepsilon \rightarrow 0$, $u_{\mathrm{N}}$ asymptotically approaches its maximum value, 2 , and $h_{\mathrm{N}}$ asymptotically approaches 0 . For sufficiently small $\varepsilon$, the solution converges to that in the limit as $u_{\mathrm{N}} \rightarrow 2$ and $h_{\mathrm{N}} \rightarrow 0$, and it becomes insensitive to the value of $\varepsilon$ (see Fig. 5a). Indeed, as shown in Fig. 8b, we can confirm that the result of the $A B$ model with a very small $\varepsilon\left(\varepsilon=1.0 \times 10^{-10}\right)$ is indistinguishable from that for $\rho_{\mathrm{c}} / \rho_{\mathrm{a}}=1000\left(\varepsilon=4.58 \times 10^{-7}\right)$. According to Fig. 5, the results of the $\mathrm{AB}$ model for the dam-break problem are insensitive to $\varepsilon$ when $\varepsilon \lesssim 10^{-5}$, which corresponds to $\rho_{\mathrm{c}} / \rho_{\mathrm{a}} \gtrsim 10^{2}$ (Fig. $5 \mathrm{~b}$ ). Consequently, we suggest that the $\mathrm{AB}$ model is favorable for simulating gravity currents with high $\rho_{\mathrm{c}} / \rho_{\mathrm{a}}$ for which the dynamics of the current is insensitive to the assumed value of $\varepsilon$.
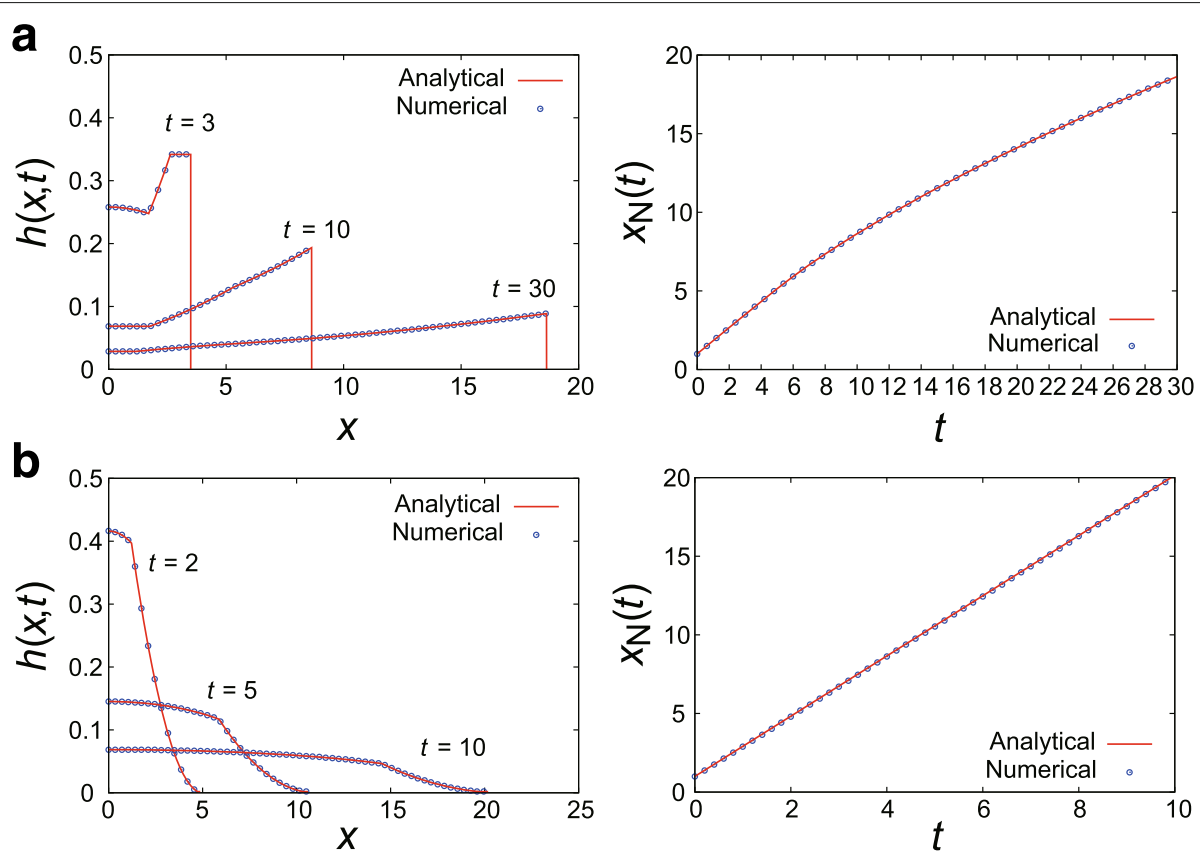

Fig. 6 Analytical results (red curves) and numerical results from the BC model (blue symbo/s) for $h(x, t)$ and $x_{N}(t)$. a $\rho_{c} / \rho_{a}=1.01 . \mathbf{b} \rho_{c} / \rho_{a}=1000$ 


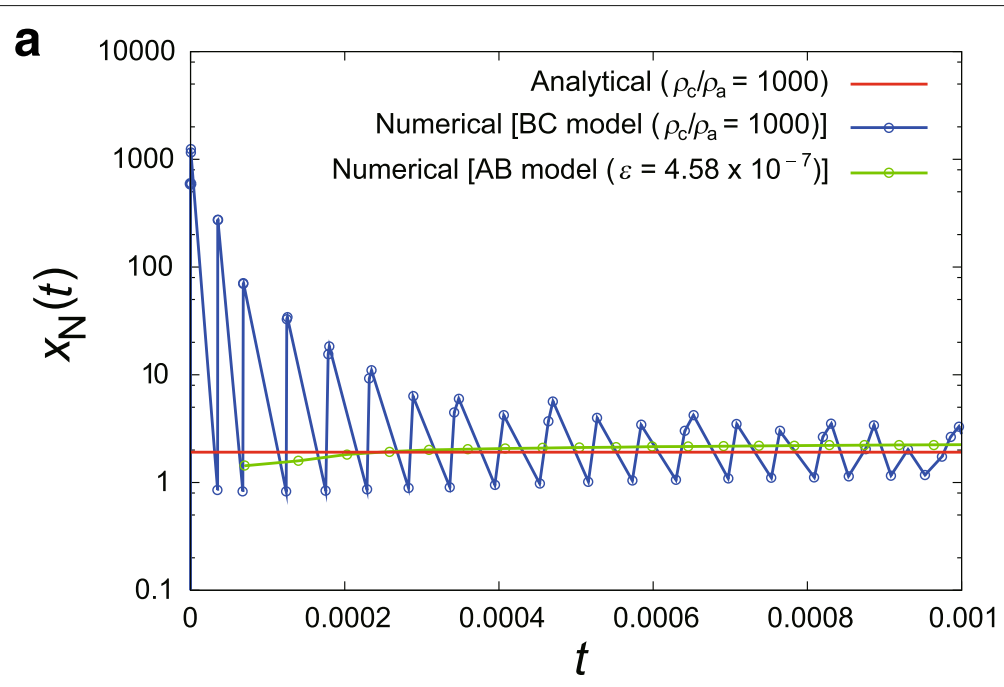

\section{b Analytical result}
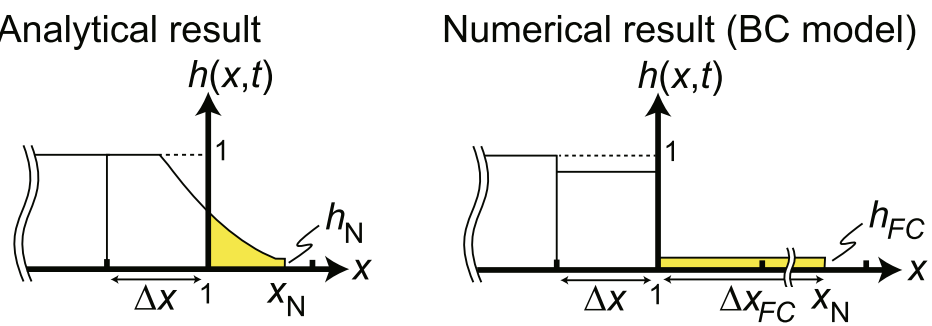

Fig. 7 Speed of the front position, $\dot{x}_{N}$, in the early time steps. a Comparisons between the analytical result with $\rho_{\mathrm{c}} / \rho_{\mathrm{a}}=1000$ (red line) and numerical results of the $\mathrm{BC}$ model with $\rho_{\mathrm{c}} / \rho_{\mathrm{a}}=1000$ (blue symbo/s) and of the $\mathrm{AB}$ model with $\varepsilon=4.58 \times 10^{-7}$ (green symbols). In the numerical calculations, $\Delta x=1.0 \times 10^{-4}$. b lllustrations of the overestimation of $\dot{x}_{N}$ by the $B C$ model in $t \lesssim 0.0002$ in (a)
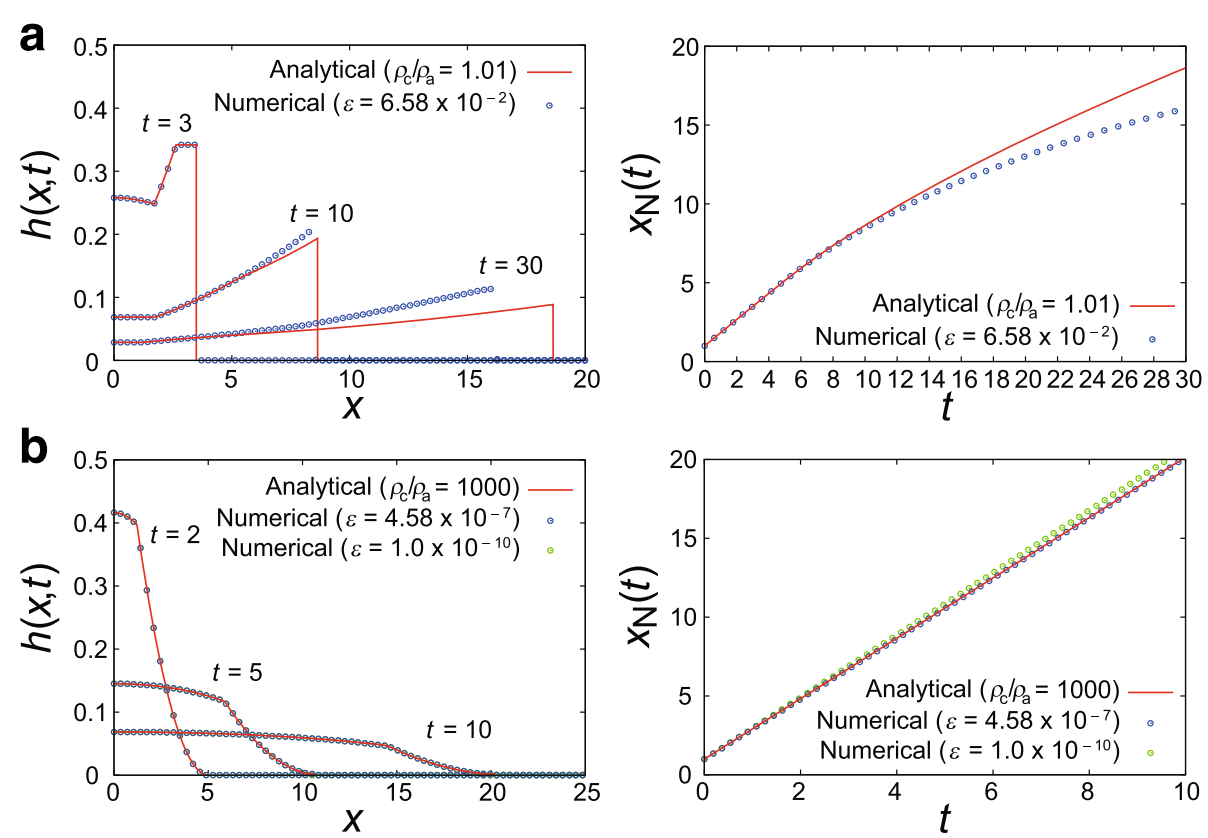

Fig. 8 Analytical results (red curves) and numerical results of the AB model (symbo/s) for $h(x, t)$ and $x_{\mathrm{N}}(t)$. a $\rho_{\mathrm{c}} / \rho_{\mathrm{a}}=1.01 . \mathbf{b} \rho_{\mathrm{c}} / \rho_{\mathrm{a}}=1000$. In the numerical results, blue symbols represent the numerical results given $\varepsilon$ based on the analytical solution during the slumping stage $\left(\mathbf{a} \varepsilon=6.58 \times 10^{-2}\right.$; b $\varepsilon=4.58 \times 10^{-7}$ ). Green symbols represent the numerical results given $\varepsilon=1.0 \times 10^{-10}$ 


\section{Applicability of the $B C$ and $A B$ models}

Our results indicate that the $\mathrm{BC}$ and $\mathrm{AB}$ models each have their own advantages and disadvantages. The results obtained from the $\mathrm{BC}$ model agree well with the analytical results when $\rho_{\mathrm{c}} / \rho_{\mathrm{a}} \lesssim 10^{2}$ (Fig. 6a), whereas they show a numerical oscillation at the flow front and tend to overestimate the front speed when $\rho_{\mathrm{c}} / \rho_{\mathrm{a}} \gtrsim 10^{2}$ (Fig. 7). No such numerical oscillation nor overestimation is observed in the results from the $\mathrm{AB}$ model. For currents with $\rho_{\mathrm{c}} / \rho_{\mathrm{a}} \gtrsim 10^{2}$, the $\mathrm{AB}$ model provides good approximations of the analytical results, given a sufficiently small $\varepsilon$ (Figs. 5 and $8 \mathrm{~b}$ ). For currents with $\rho_{\mathrm{c}} / \rho_{\mathrm{a}} \lesssim 10^{2}$, however, the $\mathrm{AB}$ model may fail to reproduce the analytical results for currents where the height and speed of the front change with time (Fig. 8a). Accordingly, we propose that the $\mathrm{BC}$ model should be used for currents with $\rho_{\mathrm{c}} / \rho_{\mathrm{a}} \lesssim$ $10^{2}$ and the $\mathrm{AB}$ model is applicable only to currents with $\rho_{\mathrm{c}} / \rho_{\mathrm{a}} \gtrsim 10^{2}$.

Because of its simple coding and numerical stability, the $\mathrm{AB}$ model with an arbitrarily small $\varepsilon$ is commonly used for simulations of gravity currents in many geophysical situations (e.g., Denlinger and Iverson 2004; Doyle et al. 2007, 2008, 2011; Larrieu et al. 2006). This model would be applicable in simulating gravity currents with high values of $\rho_{\mathrm{c}} / \rho_{\mathrm{a}}$, such as debris flows (e.g., Denlinger and Iverson 2004). However, our results suggest that it may provide inaccurate results for gravity currents with $\rho_{\mathrm{c}} / \rho_{\mathrm{a}} \lesssim 10^{2}$, such as turbidity currents and dilute pyroclastic density currents. Numerical results for $\rho_{\mathrm{c}} / \rho_{\mathrm{a}}=10$ show that the problem arises mainly from the behavior of the flow front (Fig. 9). Generally, a gravity current with a relatively low value of $\rho_{\mathrm{c}} / \rho_{\mathrm{a}}$ is characterized by the formation of a large front height, which is caused by the resistance of the ambient fluid. This large front height is successfully reproduced by the BC model (Fig. 9a), while the $\mathrm{AB}$ model fails to capture it. The results from the $\mathrm{AB}$ model with $\varepsilon=10^{-10}$ (Fig. 9b) show that the resistance at the front is too small to develop a large front height; consequently, the flow speed is substantially overestimated.

\section{Geophysical application to pyroclastic density currents}

Pyroclastic density currents (PDCs) are characterized by strong density stratification due to particle settling (e.g., Branney and Kokelaar 2002), whereby a dilute gravity current (particle suspension flow) with $\rho_{\mathrm{c}} / \rho_{\mathrm{a}}=10^{0}-10^{1}$ overrides the dense basal gravity current (fluidized granular flow) with $\rho_{\mathrm{c}} / \rho_{\mathrm{a}}=10^{2}-10^{3}$. The dynamics of PDCs is complex because the dilute and dense currents are influenced by a number of physical processes such as particle settling (e.g., Bonnecaze et al. 1993), entrainment of ambient air (e.g., Johnson and Hogg 2013; Sher and Woods 2015), and basal resistance (e.g., Roche et al. 2008). In addition to the effects of these processes, our results suggest that the application of the correct numerical model to the flow front is important if we are to understand the dynamics and sedimentation of PDCs. The BC model should be applied to the overlying dilute current, while the $\mathrm{AB}$ model is applicable to the underlying dense current. Here, we discuss how the resistance at the flow front of the dilute part influences the dynamics of PDCs as a whole.

Before discussing the complex dynamics of PDCs with strong density stratification, we briefly assess the effects of some important physical processes on the dilute and dense currents. Figure 10 shows the results of simulations using the $\mathrm{BC}$ model for a dilute gravity current generated by an instantaneous release (i.e., the dam-break problem) of an initially homogeneous particle suspension with $\rho_{\mathrm{c}} / \rho_{\mathrm{a}}=8.495$. This model accounts for the effects of particle settling and entrainment of the ambient fluid following Bonnecaze et al. (1993) and Johnson and Hogg (2013), respectively. As with a homogeneous gravity current with a low density ratio (Fig. 6a), a thick front develops in the dilute gravity current of the particle suspension, suggesting that the resistance of the ambient fluid at the front plays a significant role in the dynamics of the dilute part of PDCs regardless of the presence or absence of the effects of particle settling or entrainment.

Figure 11 compares our numerical results obtained using the $\mathrm{AB}$ model with experimental results for a
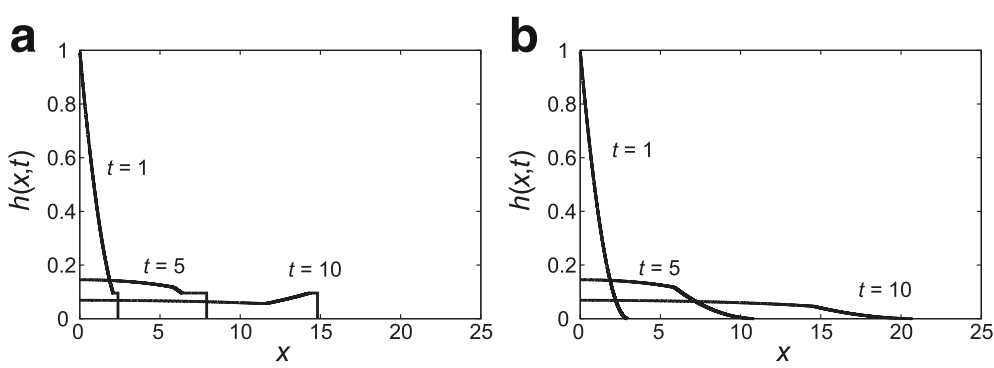

Fig. 9 Numerical results of $h(x, t)$ for $\rho_{\mathrm{c}} / \rho_{\mathrm{a}}=10$. a BC model. $\mathbf{b} \mathrm{AB}$ model with $\varepsilon=1.0 \times 10^{-10} . \ln (\mathbf{b}), \rho_{\mathrm{c}} / \rho_{\mathrm{a}}=10$ is given when the basic equations (Eqs. (1) and (2)) are non-dimensionalized using Eqs. (4) and (5) 

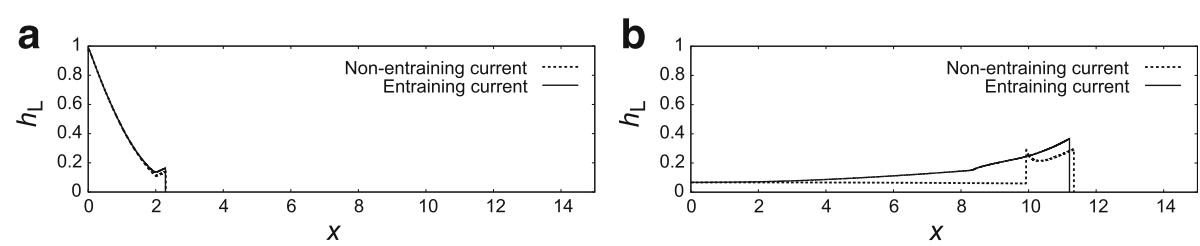

Fig. 10 Numerical results of a one-layer dilute PDC model for the dam-break problem. Heights $h$ at $\mathbf{a} t=1$ and $\mathbf{b} t=10$ are shown for cases of entraining (solid curves) and non-entraining (dotted curves) currents. The initial density ratio $\left(\rho_{\mathrm{L}}^{*} / \rho_{\mathrm{a}}\right)$ is set to 8.495 , and the BC model is applied. The variables are non-dimensionalized using the initial values (e.g., Eqs. (4) and (5)). Given parameters: aspect ratio (initial height/initial length) $=1$; initial particle concentration $=0.005$; density ratio (particles/air) $=1500$; density ratio (volcanic gas/air) $=1$; and non-dimensional particle settling speed $=0.05$

granular flow with $\rho_{\mathrm{c}} / \rho_{\mathrm{a}}=10^{2}-10^{3}$ generated by an instantaneous release of an initially fluidized bed (Roche et al. 2008). Because the initial acceleration regime of the experimental setup (i.e., just after the release; (i) in Fig. 11b) is beyond the applicable range of the shallowwater model, we focus on the subsequent regimes here. The numerical results obtained using the $\mathrm{AB}$ model show the formation of a wedge-shaped flow front (Fig. 11a), which is in agreement with the experimental results of Roche et al. (2008). The results also indicate that the dynamics of the dense fluidized granular flow can be quantitatively simulated by the $A B$ model when a suitable rheological model is applied for the basal resistance (Fig. 11b). The shear drag model explains the behavior during the constant velocity regime ((ii) in Fig. 11b; see Roche et al. 2008), whereas the Coulomb friction model better reproduces the features of the stopping regime ((iii) in Fig. 11b; see Roche et al. 2008).

The interplay between the dilute and dense parts may be crudely simulated by a two-layer model comprising a dilute layer and a dense layer (Doyle et al. 2008, 2011).
The previous two-layer models for PDCs apply the $\mathrm{AB}$ model with a small $\varepsilon$ to both layers. Here, we follow Doyle et al. (2011) for the basic formulation of the two-layer system, but apply the $\mathrm{BC}$ model to the dilute layer and the $\mathrm{AB}$ model to the dense layer. We also consider the effects of basal shear drag in the dense layer and entrainment of ambient air into the dilute layer (see Appendix for details). Figure 12 shows a representative result of our two-layer model for a PDC generated by the instantaneous release of an initially homogeneous dilute particle suspension with $\rho_{\mathrm{c}} / \rho_{\mathrm{a}}=8.495$. When the $\mathrm{BC}$ model is applied, a thick front head develops in the dilute gravity current because of the resistance of the ambient fluid at the front (see Fig. 10). On the other hand, a dense gravity current with $\rho_{\mathrm{c}} / \rho_{\mathrm{a}}=600.6$ is generated by particle settling from the overlying dilute gravity current. Because the rate at which particles are supplied to the dense layer is controlled by the conditions of the overlying dilute layer (e.g., thickness and particle concentration), the evolution and dynamics of the dense layer are critically dependent on those of the dilute layer. This suggests that the behavior of the flow
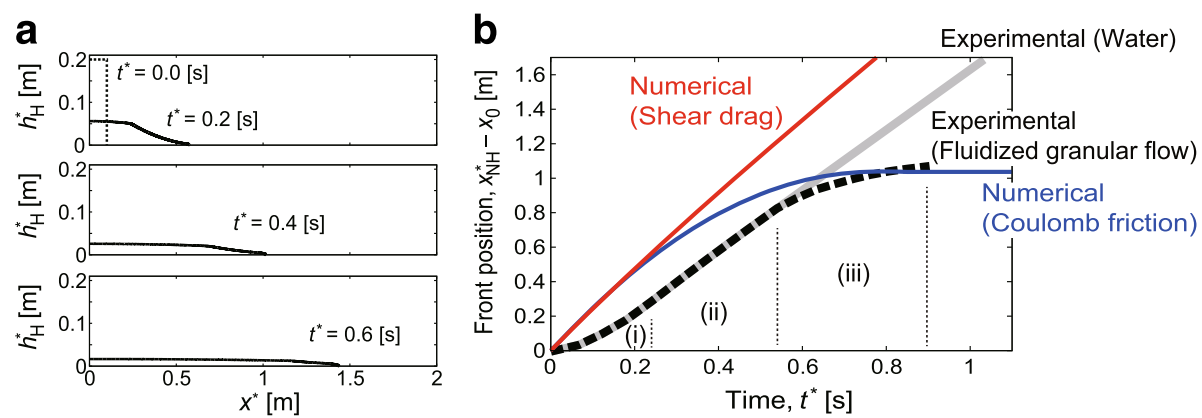

Fig. 11 Numerical results of a one-layer dense PDC model for the dam-break problem. The density ratio $\left(\rho_{H} / \rho_{\mathrm{a}}\right)$ is set to 600.6 , and the AB model with $\varepsilon=10^{-10}$ is applied. Given parameters: initial height $h_{0}=0.2 \mathrm{~m}$, initial length $x_{0}=0.1 \mathrm{~m}$, density of particles $=1500 \mathrm{~kg} / \mathrm{m}^{3} ;$ density of volcanic gas $=1 \mathrm{~kg} / \mathrm{m}^{3}$; and density of air $=1 \mathrm{~kg} / \mathrm{m}^{3}$. a Heights $h_{H}^{*}$ of the dense PDC with shear drag at $t^{*}=0.0,0.2,0.4$, and $0.6 \mathrm{~s}$. b Front positions of the dense PDC with shear drag (red solid curve) and Coulomb friction (blue solid curve). In (b), the numerical results are compared with the experimental results of an initially fluidized granular flow (black dashed curve) and water (gray solid curve) (Roche et al. 2008). The experimental result of the initially fluidized granular flow has three distinct regimes: (i) initial acceleration, (ii) constant velocity, and (iii) stopping (see Roche et al. 2008 for details). Note that during (i) and (ii), the initially fluidized granular flow behaves as water. The numerical result with shear drag reproduces the slope (i.e., the constant velocity) of (ii). The numerical result with Coulomb friction reproduces the features of (iii) 

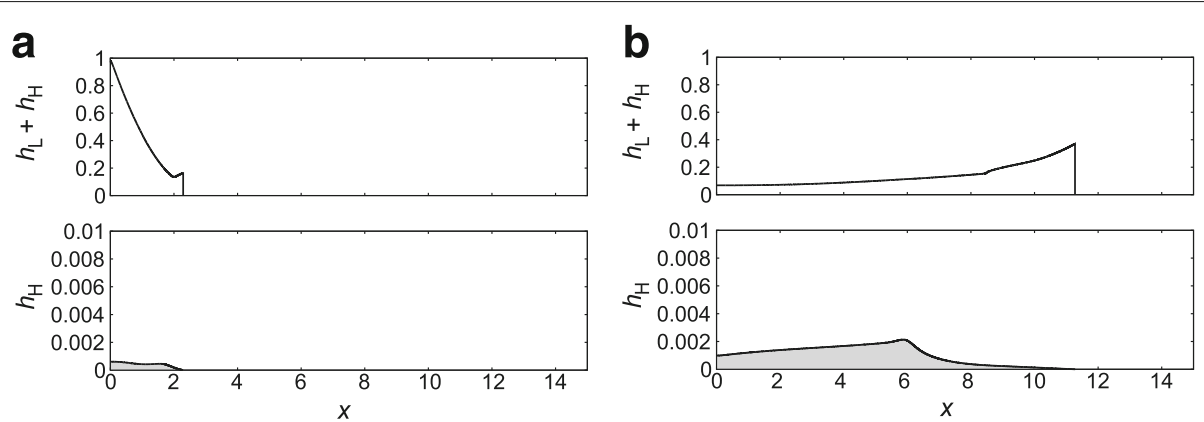

Fig. 12 Numerical results of a two-layer PDC model for the dam-break problem. The dense current height $\left(h_{\mathrm{H}}\right)$ and dilute current height $\left(h_{\mathrm{L}}+h_{\mathrm{H}}\right)$ at $\mathbf{a} t=1$ and $\mathbf{b} t=10$ are shown. A PDC is generated by the instantaneous release of an initially homogeneous dilute layer. The overlying dilute layer (initially $\rho_{\mathrm{b}}^{*} / \rho_{\mathrm{a}}=8.495$ ) is calculated using the BC model, and the underlying dense layer $\left(\rho_{\mathrm{H}} / \rho_{\mathrm{a}}=600.6\right)$ is calculated using the AB model with $\varepsilon=10^{-10}$. Shear drag is applied for the basal resistance of the dense layer. The variables are non-dimensionalized using the initial values of the dilute layer (e.g., Eqs. (4) and (5)). Given parameters: aspect ratio (initial height/initial length) = 1; initial particle concentration of the dilute layer $=0.005$; density ratio (particles/air) $=1500$; density ratio (volcanic gas/air) $=1$; and the non-dimensional particle settling speed $=0.05$

front of the dilute layer controls not only the dynamics of the dilute layer but also the dynamics of the stratified PDC as a whole.

The results in Fig. 12 are preliminary, and a comprehensive understanding of the dynamics of PDCs should consider many other effects, such as the expansion of entrained air due to heating by pyroclasts, density stratification inside the overlying dilute layer, diffusion of the pore pressure and entrainment of air in the underlying dense layer, and the transport of particles from the underlying dense layer to the overlying dilute layer (e.g., Andrews 2014; Breard and Lube 2017; Bursik and Woods 1996; Dufek and Bergantz 2007; Esposti Ongaro et al. 2016; Ishimine 2005; Roche et al. 2008; Wilson and Walker 1982). Nevertheless, preliminary results (not shown here) have already indicated the diversity of the interplay between the dilute and dense layers, which depends on the initial particle concentration and grain size. The interaction also influences the sedimentation process from the PDCs (Fujii and Nakada 1999). A systematic parametric study of the two-layer PDC model using the $\mathrm{BC}$ model is in progress, with the aim of accounting for the diversity of PDC deposits.

\section{Conclusion}

A numerical shallow-water model of simulating gravity currents for a wide range of $\rho_{\mathrm{c}} / \rho_{\mathrm{a}}$ has been proposed. In the model, the effects of varying $\rho_{\mathrm{c}} / \rho_{\mathrm{a}}$ are taken into account via the front condition. We have assessed two types of numerical models for the front condition (the Boundary Condition (BC) model and the Artificial Bed (AB) model) by comparing their numerical results with the analytical results. The results from the $\mathrm{BC}$ model agree well with the analytical results when $\rho_{\mathrm{c}} / \rho_{\mathrm{a}} \lesssim 10^{2}$. In contrast, the $\mathrm{AB}$ model generates good approximations of the analytical results for $\rho_{\mathrm{c}} / \rho_{\mathrm{a}} \gtrsim 10^{2}$. On the basis of these results, we have developed a two-layer model of pyroclastic density currents (PDCs), in which the BC model is used for the overlying dilute part $\left(\rho_{\mathrm{c}} / \rho_{\mathrm{a}}=\right.$ $\left.10^{0}-10^{1}\right)$ and the $A B$ model is used for the underlying dense part $\left(\rho_{\mathrm{c}} / \rho_{\mathrm{a}}=10^{2}-10^{3}\right)$. This two-layer model successfully simulates some essential features of PDCs with strong density stratification.

\section{Appendix: two-layer model of pyroclastic density currents}

The dynamics of PDCs in which a strong density stratification develops is described here by a two-layer shallowwater model with overlying dilute and underlying dense layers (Fig. 13). The dilute layer is modeled as a dilute particle suspension, which is a continuum where monodisperse solid particles are suspended in an incompressible gas phase (volcanic gas and entrained air). Its vertically averaged mass and momentum conservation equations are respectively

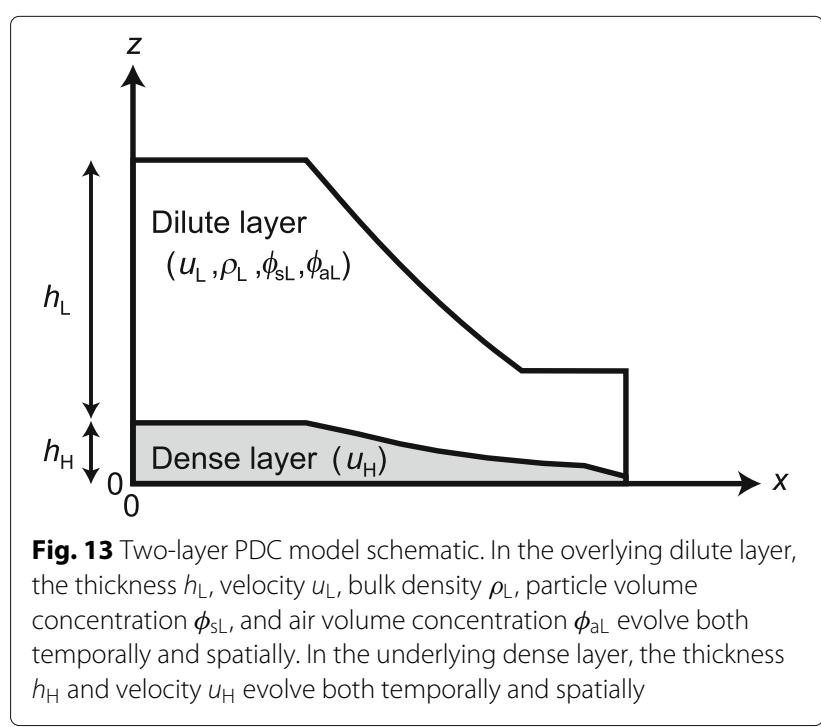




$$
\begin{gathered}
\frac{\partial}{\partial t^{*}}\left(\rho_{\mathrm{L}}^{*} h_{\mathrm{L}}^{*}\right)+\frac{\partial}{\partial x^{*}}\left(\rho_{\mathrm{L}}^{*} u_{\mathrm{L}}^{*} h_{\mathrm{L}}^{*}\right)=\rho_{\mathrm{a}} E\left|u_{\mathrm{L}}^{*}\right|-\rho_{\mathrm{H}} \frac{\phi_{\mathrm{sL}}}{\phi_{\mathrm{sH}}} W_{\mathrm{s}}, \\
\frac{\partial}{\partial t^{*}}\left(\rho_{\mathrm{L}}^{*} u_{\mathrm{L}}^{*} h_{\mathrm{L}}^{*}\right)+\frac{\partial}{\partial x^{*}}\left(\rho_{\mathrm{L}}^{*} u_{\mathrm{L}}^{* 2} h_{\mathrm{L}}^{*}+\frac{\rho_{\mathrm{L}}^{*}-\rho_{\mathrm{a}}}{2} g h_{\mathrm{L}}^{* 2}\right) \\
=-\left(\rho_{\mathrm{L}}^{*}-\rho_{\mathrm{a}}\right) g h_{\mathrm{L}}^{*} \frac{\partial h_{\mathrm{H}}^{*}}{\partial x^{*}}-\rho_{\mathrm{H}} \frac{\phi_{\mathrm{sL}}}{\phi_{\mathrm{sH}}} u_{\mathrm{L}}^{*} W_{\mathrm{s}}-\tau_{\mathrm{m}}^{*} .
\end{gathered}
$$

The dense layer, modeled as a fluidized granular flow consisting of solid particles and volcanic gas, has the following vertically averaged mass and momentum conservation equations:

$$
\begin{aligned}
& \frac{\partial h_{\mathrm{H}}^{*}}{\partial t^{*}}+\frac{\partial}{\partial x^{*}}\left(u_{\mathrm{H}}^{*} h_{\mathrm{H}}^{*}\right)=\frac{\phi_{\mathrm{sL}}}{\phi_{\mathrm{sH}}} W_{\mathrm{s}}, \\
& \frac{\partial}{\partial t^{*}}\left(u_{\mathrm{H}}^{*} h_{\mathrm{H}}^{*}\right)+\frac{\partial}{\partial x^{*}}\left(u_{\mathrm{H}}^{* 2} h_{\mathrm{H}}+\frac{1}{2} \frac{\rho_{\mathrm{H}}-\rho_{\mathrm{a}}}{\rho_{\mathrm{H}}} g h_{\mathrm{H}}^{* 2}\right) \\
& \quad=-\frac{\tau_{\mathrm{b}}^{*}}{\rho_{\mathrm{H}}}+\frac{\tau_{\mathrm{m}}^{*}}{\rho_{\mathrm{H}}}+\frac{\phi_{\mathrm{sL}}}{\phi_{\mathrm{sH}}} u_{\mathrm{L}}^{*} W_{\mathrm{s}}-\frac{h_{\mathrm{H}}^{*}}{\rho_{\mathrm{H}}} \frac{\partial}{\partial_{x^{*}}}\left(\left(\rho_{\mathrm{L}}^{*}-\rho_{\mathrm{a}}\right) g h_{\mathrm{L}}^{*}\right) .
\end{aligned}
$$

Here, $\phi\left(x^{*}, t^{*}\right)$ is volumetric concentration, and the subscripts $\mathrm{L}, \mathrm{H}, \mathrm{s}, \mathrm{g}$, and a denote the dilute (i.e., low-particle concentration) and dense (i.e., high-particle concentration) layers, solid particles, volcanic gas, and air, respectively. $W_{\mathrm{s}}$ is the settling velocity of the particles from the base of the dilute layer, $E$ is the entrainment coefficient, $\tau_{\mathrm{m}}^{*}$ is the interfacial shear drag, and $\tau_{\mathrm{b}}^{*}$ is basal resistance of the dense layer.

The bulk density of the dilute layer is denoted by $\rho_{\mathrm{L}}^{*}=$ $\rho_{\mathrm{s}} \phi_{\mathrm{sL}}+\rho_{\mathrm{a}} \phi_{\mathrm{aL}}+\rho_{\mathrm{g}}\left(1-\phi_{\mathrm{SL}}-\phi_{\mathrm{aL}}\right)$, in which the volume concentrations of the solid particles, $\phi_{\mathrm{sL}}$, and of the entrained air, $\phi_{\mathrm{aL}}$, evolve both temporally and spatially on the basis of their mass conservation equations:

$$
\begin{aligned}
& \frac{\partial}{\partial t^{*}}\left(\phi_{\mathrm{SL}} h_{\mathrm{L}}^{*}\right)+\frac{\partial}{\partial x^{*}}\left(\phi_{\mathrm{sL}} u_{\mathrm{L}}^{*} h_{\mathrm{L}}^{*}\right)=-\phi_{\mathrm{sL}} W_{\mathrm{s}}, \\
& \frac{\partial}{\partial t^{*}}\left(\phi_{\mathrm{aL}} h_{\mathrm{L}}^{*}\right)+\frac{\partial}{\partial x^{*}}\left(\phi_{\mathrm{aL}} u_{\mathrm{L}}^{*} h_{\mathrm{L}}^{*}\right)=E\left|u_{\mathrm{L}}^{*}\right| .
\end{aligned}
$$

In the dilute layer, it is assumed that turbulent mixing is sufficiently intense to maintain vertically uniform volumetric concentrations (e.g., Bonnecaze et al. 1993; Bursik and Woods 1996; Johnson and Hogg 2013). The dense layer is assumed to have a constant bulk density $\rho_{\mathrm{H}}=\rho_{\mathrm{s}} \phi_{\mathrm{sH}}+\rho_{\mathrm{g}}\left(1-\phi_{\mathrm{sH}}\right)$, where the particle volumetric concentration $\phi_{\mathrm{sH}}$ is set to 0.4 (Breard et al. 2016).

Interactions between the two layers are treated in the source terms of Eqs. (21)-(26) (i.e., the right-hand sides of the equations). Particle settling from the dilute layer to the dense layer is taken into account in the second source terms in Eqs. (21) and (22), the source terms in Eqs. (23) and (25), and the third source term in Eq. (24). The acceleration of the dilute layer over the basal contact is taken into account in the first source term in Eq. (22). The pressure gradient on the dense layer exerted by variations in the height of the dilute layer is taken into account in the fourth source term in Eq. (24).

The entrainment of ambient air into the dilute layer is taken into account in the first source term in Eq. (21) and the source term in Eq. (26). Thermal expansion of the entrained air is neglected here for the sake of ease. Air entrainment is also assumed to occur on the upper surface of the dilute layer (e.g., Bursik and Woods 1996; Johnson and Hogg 2013), although a different process for entrainment was recently proposed by Sher and Woods (2015). We adopted the entrainment coefficient proposed by Johnson and $\operatorname{Hogg}$ (2013): i.e., $E=0.075 /(1+27 R i)$, where $R i \equiv\left(\rho_{\mathrm{L}}^{*}-\rho_{\mathrm{a}}\right) g h_{\mathrm{L}}^{*} /\left(\rho_{\mathrm{L}}^{*} u_{\mathrm{L}}^{* 2}\right)$

The interfacial drag $\tau_{\mathrm{m}}^{*}$ and the basal resistance of the dense layer $\tau_{\mathrm{b}}^{*}$ are treated in the source terms in Eqs. (22) and (24). The interfacial shear drag $\tau_{\mathrm{m}}^{*}$ is given by (Doyle et al. 2008, 2011):

$$
\tau_{\mathrm{m}}^{*}=\rho_{\mathrm{L}}^{*} C_{\mathrm{d}}\left(u_{\mathrm{L}}^{*}-u_{\mathrm{H}}^{*}\right)\left|u_{\mathrm{L}}^{*}-u_{\mathrm{H}}^{*}\right|,
$$

where the drag coefficient $C_{\mathrm{d}}$ is set to 0.001 (Hogg and Pritchard 2004). The basal resistance $\tau_{\mathrm{b}}^{*}$ is modeled such that shear drag is adopted during the constant velocity regime ((ii) of Fig. 11b) and Coulomb friction is adopted during the stopping regime ((iii) of Fig. 11b):

$\tau_{\mathrm{b}}^{*}= \begin{cases}\rho_{\mathrm{H}} C_{\mathrm{d}} u_{\mathrm{H}}^{*}\left|u_{\mathrm{H}}^{*}\right| & \text { (Shear drag), } \\ \tan \delta\left(\rho_{\mathrm{H}}-\rho_{\mathrm{a}}\right) g h_{\mathrm{H}}^{*} u_{\mathrm{H}}^{*} /\left|u_{\mathrm{H}}^{*}\right| & \text { (Coulomb friction), }\end{cases}$

(Figure 11b; see Roche et al. 2008 for details), where the dynamic basal friction angle $\delta$ is set to $20^{\circ}$ (Doyle et al. 2008).

The front condition of the dilute layer is given by (Ungarish 2007):

$$
u_{\mathrm{NL}}^{*}=F r \sqrt{\frac{\rho_{\mathrm{NL}}^{*}-\rho_{\mathrm{a}}}{\rho_{\mathrm{a}}} g h_{\mathrm{NL}}^{*}} \quad \text { at } \quad x^{*}=x_{\mathrm{NL}}^{*},
$$

which is numerically treated by the $\mathrm{BC}$ model. The front condition of the dense layer, given by Eq. (3), is numerically treated by the $\mathrm{AB}$ model with $\varepsilon=10^{-10}$.

In calculating the two-layer PDC model (Fig. 12), the conservation Eqs. (21)-(26) were numerically solved. In calculating the one-layer dilute PDC model (Fig. 10), we solved conservation Eqs. (21), (22), (25), and (26), where $h_{\mathrm{H}}^{*} \approx 0$ and $u_{\mathrm{H}}^{*} \approx 0$. In calculating the one-layer dense PDC model (Fig. 11), we solved conservation Eqs. (23) and (24). For numerical simulations, we used a fractional-step method to solve the conservation equations with source terms (e.g., LeVeque 2002), and the HLL approximate Riemann solver to calculate the intercell flux of the equations (e.g., Toro 2009).

\section{Abbreviations}

AB model: Artificial Bed model; BC model: Boundary Condition model; PDC: Pyroclastic density current 


\section{Acknowledgements}

We thank Editor Colin J. N. Wilson, Reviewer Eric C. P. Breard, and an anonymous reviewer for their thoughtful comments.

\section{Funding}

Not applicable.

\section{Authors' contributions}

HAS carried out this study under the supervision of TK and YJS and prepared the first draft of the manuscript. All authors contributed to writing the manuscript and approved the final version.

\section{Authors' information}

HAS is a Ph.D. candidate supervised by TK and YJS. TK is a Professor at the Earthquake Research Institute, the University of Tokyo. YJS is an Assistant Professor at the Earthquake Research Institute, the University of Tokyo.

\section{Competing interests}

The authors declare that they have no competing interests.

\section{Publisher's Note}

Springer Nature remains neutral with regard to jurisdictional claims in published maps and institutional affiliations.

\section{Received: 21 July 2016 Accepted: 3 March 2017}

Published online: 28 March 2017

\section{References}

Andrews BJ (2014) Dispersal and air entrainment in unconfined dilute pyroclastic density currents. Bull Volcanol 76:1-14 doi:10.1007/s00445-014-0852-4

Benjamin TB (1968) Gravity currents and related phenomena. J Fluid Mech 31:209-48. doi:10.1017/S0022112068000133

Birman V, Martin J, Meiburg E (2005) The non-boussinesq lock-exchange problem. part 2. High-resolution simulations. J Fluid Mech 537:125-44. doi:10.1017/S0022112005005033

Bonnecaze RT, Huppert HE, Lister JR (1993) Particle-driven gravity currents. J Fluid Mech 250:339-69. doi:10.1017/S002211209300148X

Bonometti T, Balachandar S (2010) Slumping of non-boussinesq density currents of various initial fractional depths: a comparison between direct numerical simulations and a recent shallow-water model. Comput Fluids 39:729-34

Bonometti T, Ungarish M, Balachandar S (2011) A numerical investigation of high-reynolds-number constant-volume non-boussinesq density currents in deep ambient. J Fluid Mech 673:574-602. doi:10.1017/S0022112010006506

Branney MJ, Kokelaar BP (2002) Pyroclastic density currents and the sedimentation of ignimbrites. Geol Soc, London

Breard ECP, Lube G (2017) Inside pyroclastic density currents-uncovering the enigmatic flow structure and transport behaviour in large-scale experiments. Earth Planet Sci Lett 458:22-36. doi:10.1016/j.epsl.2016.10.016

Breard ECP, Lube G, Jones JR, Dufek J, Cronin SJ, Valentine GA, Moebis A (2016) Coupling of turbulent and non-turbulent flow regines within pyroclastic density currents. Nat Geosci 9:767-71. doi:10.1038/NGEO2794

Bursik MI, Woods AW (1996) The dynamics and thermodynamics of large ash flows. Bull Volcanol 58:175-193. doi:10.1007/s004450050134

Cantero MI, Lee J, Balachandar S, Garcia MH (2007) On the front velocity of gravity currents. J Fluid Mech 586:1-39. doi:10.1017/S0022112007005769

Denlinger RP, Iverson RM (2004) Granular avalanches across irregular three-dimensional terrain: 1. theory and computation. J Geophys Res 109:F01014. doi:10.1029/2003JF000085

Doyle EE, Hogg AJ, Mader HM, Sparks RSJ (2008) Modeling dense pyroclastic basal flows from collapsing columns. Geophys Res Lett 35:L04305. doi:10.1029/2007GL032585

Doyle EE, Hogg AJ, Mader HM (2011) A two-layer approach to modelling the transformation of dilute pyroclastic currents into dense pyroclastic flows. Proc R Soc A 467:1348-71. doi:10.1098/rspa.2010.0402

Doyle EE, Huppert HE, Lube G, Mader HM, Sparks RSJ (2007) Static and flowing regions in granular collapses down channels: insights from a sedimenting shallow-water model. Phys Fluids 106601:19. doi:10.1063/1.2773738
Dressler RF (1954) Comparison of theories and experiments for the hydraulic dam-break wave. Int Assoc Sci Hydrol 3:319-28

Dufek J, Bergantz G (2007) Suspended load and bed-load transport of particle-laden gravity currents: the role of particle-bed interaction. Theor Comput Fluid Dyn 21:119-45. doi:10.1007/s00162-007-0041-6

Esposti Ongaro T, Orsucci S, Cornolti F (2016) A fast, calibrated model for pyroclastic density currents kinematics and hazard. J Volcanol Geotherm Res 327:257-72. doi:10.1016/j.jvolgeores.2016.08.002

Fujii T, Nakada S (1999) The 15 September 1991 pyroclastic flows at Unzen Volcano (Japan): a flow model for associated ash-cloud surges. J Volcanol Geotherm Res 89:159-72. doi:10.1016/S0377-0273(98)00130-9

Gröbelbauer H, Fanneløp T, Britter R (1993) The propagation of intrusion fronts of high density ratios. J Fluid Mech 250:669-87. doi:10.1017/ S0022112093001612

Hallworth MA, Huppert HE (1998) Abrupt transitions in high-concentration, particle-driven gravity currents. Phys Fluids 10:1083. doi:10.1063/ 1.869633

Härtel C, Meiburg E, Necker F (2000) Analysis and direct numerical simulation of the flow at a gravity-current head. part 1. flow topology and front speed for slip and no-slip boundaries. J Fluid Mech 418:189-212. doi:10.1017/S0022112000001221

Hogg AJ (2006) Lock-release gravity currents and dam-break flows. J Fluid Mech 569:61-87. doi:10.1017/S0022112006002588

Hogg AJ, Pritchard D (2004) The effects of hydraulic resistance on dam-break and other shallow inertial flows. J Fluid Mech 501:179-212 doi:10.1017/S0022112003007468

Huppert HE, Simpson JE (1980) The slumping of gravity currents. J Fluid Mech 99:785-99. doi:10.1017/S0022112080000894

Ishimine Y (2005) Numerical study of pyroclastic surges. J Volcanol Geotherm Res 139:33-57. doi:10.1016/j.jvolgeores.2004.06.017

Iverson RM (1997) The physics of debris flows. Rev Geophys 35:245-96. doi:10.1029/97RG00426

Johnson CG, Hogg AJ (2013) Entraining gravity currents. J Fluid Mech 731:477-508. doi:10.1017/jfm.2013.329

Larrieu E, Staron L, Hinch E (2006) Raining into shallow-water as a description of the collapse of a column of grains. J Fluid Mech 554:259-70. doi:10.1017/S0022112005007974

LeVeque RJ (2002) Finite volume methods for hyperbolic problems. Cambridge University Press, Cambridge

Marino B, Thomas L, Linden P (2005) The front condition for gravity currents. J Fluid Mech 536:49-78. doi:10.1017/S0022112005004933

Martin JC, Moyce WJ (1952) An experimental study of the collapse of liquid columns on a rigid horizontal plane. Phil Trans R Soc Lond A 244:312-24. doi:10.1098/rsta.1952.0006

Meiburg E, Kneller B (2010) Turbidity currents and their deposits. Annu Rev Fluid Mech 42:135-56. doi:10.1146/annurev-fluid-121108-145618

Nield SE, Woods AW (2004) Effects of flow density on the dynamics of dilute pyroclastic density currents. J Volcanol Geotherm Res 132:269-81. doi:10.1016/S0377-0273(03)00314-7

Ooi SK, Constantinescu G, Weber L (2009) Numerical simulations of lock-exchange compositional gravity current. J Fluid Mech 635:361-88. doi:10.1017/S0022112009007599

Roche O, Montserrat S, Niño Y, Tamburrino A (2008) Experimental observations of water-like behavior of initially fluidized, dam break granular flows and their relevance for the propagation of ash-rich pyroclastic flows. J Geophys Res 113:B12203. doi:10.1029/2008JB005664

Rottman JW, Simpson JE (1983) Gravity currents produced by instantaneous releases of a heavy fluid in a rectangular channel. J Fluid Mech 135:95-110. doi:10.1017/S0022112083002979

Sher D, Woods AW (2015) Gravity currents: Entrainment, stratification and self-similarity. J Fluid Mech 784:130-62. doi:10.1017/jfm.2015.576

Stoker JJ (1992) Water waves: the mathematical theory with applications. Wiley, New York

Toro EF (2001) Shock-capturing methods for free-surface shallow flows. Wiley, Chichester

Toro, EF (2009) Riemann solvers and numerical methods for fluid dynamics: a practical introduction. Springer, Berlin

Ungarish M, Zemach T (2005) On the slumping of high reynolds number gravity currents in two-dimensional and axisymmetric configurations. European J Mech B/Fluids 24:71-90. doi:10.1016/j.euromechflu. 2004.05.006 
Ungarish M (2007) A shallow-water model for high-reynolds-number gravity currents for a wide range of density differences and fractional depths. J Fluid Mech 579:373-82. doi:10.1017/S0022112007005484

Ungarish M (2009) An introduction to gravity currents and intrusions. CRC Press, Boca Raton

Wilson CJN, Walker GPL (1982) Ignimbrite depositional facies: the anatomy of a pyroclastic flow. J Geol Soc 139:581-92. doi:10.1144/gsjgs.139.5.0581

\section{Submit your manuscript to a SpringerOpen ${ }^{\circ}$ journal and benefit from:}

- Convenient online submission

- Rigorous peer review

- Immediate publication on acceptance

- Open access: articles freely available online

- High visibility within the field

- Retaining the copyright to your article

Submit your next manuscript at $>$ springeropen.com 